\title{
Hypoxia induces lactate secretion and glycolytic efflux by downregulating mitochondrial pyruvate carrier levels in human umbilical vein endothelial cells
}

\author{
DONG WANG $^{1 *}$, QINGJIE WANG ${ }^{2}$, GAOLIANG YAN $^{1}$, YONG QIAO $^{1}$, \\ BOQIAN ZHU ${ }^{1}$, BO LIU ${ }^{1}$ and CHENGCHUN TANG ${ }^{1 *}$ \\ ${ }^{1}$ Department of Cardiology, Zhongda Hospital of Southeast University Medical School, Nanjing, Jiangsu 210009; \\ ${ }^{2}$ Department of Cardiology, Changzhou Hospital of Nanjing Medical University, Changzhou, Jiangsu 213004, P.R. China
}

Received December 16, 2017; Accepted May 15, 2018

DOI: $10.3892 / \mathrm{mmr} .2018 .9079$

\begin{abstract}
The mitochondrial pyruvate carrier (MPC) complex, located on the inner mitochondrial membrane, transports pyruvate to the mitochondrial matrix for oxidative phosphorylation. Previous studies have shown that the MPC complex is a key regulator of glycolysis in tumor cells. The present study evaluated the role of the MPC under hypoxic conditions in human umbilical vein endothelial cells, which rely on glycolysis for energy generation. It was indicated that hypoxia led to an increase in lactate secretion and a decrease in MPC1 and MPC2 levels, which were upregulated following re-oxygenation. In addition, the knockdown of MPC1 or treatment with the MPC inhibitor UK5099 increased the levels of glycolytic enzymes, HK2, PFKFB3, and LDHA, promoting glycolysis and lactate secretion. Taken together, the present data revealed that hypoxia can induce lactate secretion and glycolytic efflux by downregulating MPC levels.
\end{abstract}

\section{Introduction}

Oxidative phosphorylation (OXPHOS) is an essential metabolic pathway for the generation of energy in cells. In most eukaryotes, this process takes place in mitochondria and usually requires the cytosolic substrate pyruvate. Extensive effort has been put into identifying the molecular carrier responsible for the transport of pyruvate across the inner mitochondrial membrane (IMM) and into the mitochondrial

Correspondence to: Professor Chengchun Tang, Department of Cardiology, Zhongda Hospital of Southeast University Medical School, 87 Dingjiaqiao Road, Nanjing, Jiangsu 210009, P.R. China E-mail: tangchengchun@medmail.com.cn

*Contributed equally

Key words: mitochondrial pyruvate carrier, lactate, hypoxia, glycolysis, endothelial cells matrix. In 2012, two independent groups discovered the long sought-after mammalian mitochondrial pyruvate carrier (MPC), which is composed of two paralogous subunits, MPC1 and MPC2 $(1,2)$. The location of the MPC complex at the IMM puts it at the intersection between cytosolic glycolysis and mitochondrial OXPHOS. Subsequent works confirmed the critical role of the MPC complex in multiple cellular functions, including the metabolism of glucose and pyruvate, fibrosis, and effects on drug efficacy (3-6). Unlike other mitochondrial carriers that can function as a monomer, pyruvate transport by the MPC complex requires both MPC1 and MPC2 (1). In addition, the function of the MPC complex is closely related to mitochondrial function, especially $\mathrm{MPC} 1$, in mammals.

Studies show that MPC dysfunction causes various diseases, including lactic acidosis, hyperpyruvatemia, tumors and other severe diseases (7). As the gatekeeper for pyruvate entry into mitochondria, the MPC is thought to be of core position in cell metabolic programming. MPC dysfunction or expression reduction blocks pyruvate entry into the TCA cycle, which leads to a metabolism switch to increase glycolysis. Depending on their localization, endothelial cells (ECs) are exposed to various oxygen tensions. Like tumor cells, ECs rely on glycolysis (specifically, aerobic glycolysis) for energy production (8). Glycolysis provides bioenergetic intermediates, but generates less ATP. In physiological situations, cells change from aerobic oxidation to glycolysis in hypoxic conditions. While most pyruvate, the primary product of glycolysis, is converted to lactate by lactate dehydrogenase A (LDHA), a small amount of pyruvate is transferred to mitochondria for OXPHOS. Given that the MPC complex represents a crucial checkpoint in the regulation of cellular metabolism, understanding how it is regulated could have an enormous impact on the treatment of human diseases. Currently, whether and how MPC expression is altered in response to stressful conditions such as hypoxia is unclear. Knowledge gained from such research will advance our understanding of the roles and regulatory mechanisms of the MPC complex in ECs.

For a long time, most of the studies on human vascular ECs are based on human umbilical vein ECs (HUVECs), and all the functions of ECs can be achieved through in vitro 
culture (9). HUVECs provide a classic model system to study many aspects of endothelial function and disease, such as tumor-associated angiogenesis, cardiovascular-related complications, oxidative stress, hypoxia and inflammation related pathways in endothelia, mode of action and cardiovascular protection effects of various compounds.

In this study, we initially examined the MPC expression levels in several metabolic cell types, including HUVECs, human coronary artery ECs (HCAECs), human umbilical vein smooth muscle cells (HUSMCs), and human embryonic kidney cells 293. Our data indicate that, while MPC1 and MPC2 were expressed at significantly higher levels in 293T cells, no significant differences were observed among HUVECs, HCAECs, and HUSMCs. Furthermore, hypoxia was found to increase lactate secretion while it led to reduced MPC1 and MPC2 levels in HUVECs. Following re-oxygenation, the levels of both subunits rose. To explore the role of the MPC complex in cellular metabolism under hypoxia, a small interfering RNA (siRNA) targeting the $m p c l$ gene and the MPC inhibitor UK5099 were utilized to inhibit MPC1 expression and MPC function. Treatment with either the siRNA or UK5099 promoted aerobic glycolysis and lactate secretion in HUVECs under hypoxia. These results indicate that hypoxia can induce lactate secretion and glycolytic flux by downregulating MPC levels.

\section{Materials and methods}

Cell culture. HCAECs were purchased from Promocell (Heidelberg, Germany). HUSMCs and 293T were grown in Dulbecco's modified Eagle's minimal essential medium (DMEM) supplemented with $10 \%$ fetal bovine serum. HUVECs and HCAECs were cultured in endothelial growth media-2 (Promocell) supplemented with EC growth supplement (Promocell) at $37^{\circ} \mathrm{C}$ in a humidified atmosphere of $95 \%$ air and $5 \% \mathrm{CO}_{2}$. To determine the effect of hypoxia-normoxia transition on MPC expression, cells were incubated under hypoxia $\left(1 \% \mathrm{O}_{2}\right.$ and $\left.99 \% \mathrm{~N}_{2}\right)$ for $24 \mathrm{~h}$, and they were then cultured for $24 \mathrm{~h}$ under normoxia $\left(95 \%\right.$ air and $\left.5 \% \mathrm{CO}_{2}\right)$.

Reagents. JC-1 fluorescent probe was purchased from Beyotime Institute of Biotechnology (Jiangsu, China). UK5099 was purchased from Sigma-Aldrich (Merck KGaA, Darmstadt, Germany). UK5099 was dissolved in Dimethyl sulfoxide (DMSO), and the final concentration of DMSO was less than $0.05 \%$. UK5099 was optimized to a final concentration of $40 \mu \mathrm{M}$ to reduce pyruvate transportation into mitochondrial based on a series of UK5099 dose tested in a range of $10 \mu \mathrm{M}$ to $100 \mu \mathrm{M}$ as previously published $(10,11)$.

Silencing experiments. HUVECs were grown in 6-well plates up to $85 \%$ confluence and transfected using Lipofectamine RNAiMAX (Invitrogen, Merelbeke, Belgium) with 200 pmol of specific siRNA (GenePharma, Shanghai, China) targeting MPC1 (Accession no. NM_001270879.1) (sense: 5'-GGC UUAUCAAACACGAGAUTT-3'; antisense: 5'-AUCUCG UGUUUGAUAAGCCTT-3'). All star control siRNA (sense: 5'-UUCUUCGAACGUGUCACGUTT-3'; antisense: 5'-ACG UGACACGUUCGGAGAATT-3') was used as negative control. Silencing efficiency was detected by western blotting.
Reverse transcription-polymerase chain reaction ( $R T-P C R)$. Total RNA was isolated from cells using standard procedure according to the manufacturer's instructions. The RNA was reverse-transcribed to cDNA with random primers using All-In-One RT MasterMix (Applied Biological Materials, Inc., Richmond, $\mathrm{BC}$, Canada) at $25^{\circ} \mathrm{C}$ for $10 \mathrm{~min}, 42^{\circ} \mathrm{C}$ for $15 \mathrm{~min}$, followed by $85^{\circ} \mathrm{C}$ for $5 \mathrm{~min}$. PCR was performed using an iCycler (Bio-Rad Laboratories, Inc., Hercules, CA, USA). The reaction mixture consisted of $1 \mu \mathrm{l}$ template cDNA, $0.2 \mu \mathrm{M}$ of each primer and $25 \mu \mathrm{l} 2 \mathrm{xPCR}$ Taq MasterMix (Applied Biological Materials, Inc.). PCR was performed for 32 cycles for each gene with denaturation at $94^{\circ} \mathrm{C}$ for $30 \mathrm{sec}$, annealing at $61^{\circ} \mathrm{C}$ for $30 \mathrm{sec}$, and extension at $72^{\circ} \mathrm{C}$ for $20 \mathrm{sec}$. PCR products were quantified using NIH Image. The primer sequences purchased from Invitrogen for MPC1 (sense: 5'-GCCTACAAGGTACAGCCTCG-3'; antisense: 5'-GTG TTTGATAAGCCGCCCTC-3') and for MPC2 (Accession no. NM_001143674.3) (sense: 5'-TACCACCGGCTCCTC GATAA-3'; antisense: 5'-ACAGCAGATTGAGCTGTG CT-3'). $\beta$-actin (sense: $5^{\prime}$-CCCATCTATGAGGGTTACGC-3'; antisense: 5'-TTTAATGTCACGCACGATTTC-3') was used as a reference gene.

Quantitative PCR ( $q P C R)$. qPCR was performed in a final volume of $20 \mu \mathrm{l}$ containing cDNA template, primers and qRCR MasterMix (Applied Biological Materials, Inc.) using the 7300 qPCR system (Applied Biosystems; Thermo Fisher Scientific, Inc., Waltham, MA, USA) as described in the manufacture's manual. PCR amplification was carried out on $95^{\circ} \mathrm{C}$ for $30 \mathrm{sec}$, 40 cycles at $95^{\circ} \mathrm{C}$ for $5 \mathrm{sec}$ and $60^{\circ} \mathrm{C}$ for $31 \mathrm{sec}$ using the following primers: MPC1, MPC2 and $\beta$-actin were described above; Fis1 (Accession no. NM_016068.2) (sense: 5'-CTTAAA GTACGTCCGCGGGT-3'; antisense: 5'-GCCCACGAGTCC ATCTTTCT-3'); Opa1 (Accession no. NM_015560.2) (sense: 5'-TACCAGCCTCGCAGGAATTT-3'; antisense: 5'-CTTTTT GGCTGTGTAGCCACC-3'). The relative mRNA amounts of target genes were normalized to the values of $\beta$-actin. The results were expressed as fold-changes of Cquantification cycle (Cq) value relative to the controls using the $2^{-\Delta \Delta \mathrm{Cq}}$ method.

Western blotting. Western blotting was carried as described previously. In brief, cells lysate with equal amount of protein $(50 \mu \mathrm{g})$ were separated by $10 \%$ SDS-polyacrylamide gel electrophoresis and then transferred electronically to the polyvinylidene difluoride membranes. Membranes were blocked in 5\% non-fat milk powder in TBST for $1 \mathrm{~h}$ at room temperature, and then incubated with targeting antibodies: MPC1 (ab74871, dilution 1:1,000; Abcam, Cambridge, UK), anti-MPC2 (ab111380, dilution 1:1,000; Abcam), hexokinase II (HK2; ab104836, dilution 1:1,000; Abcam), LDHA (ab101562, dilution 1:1,000; Abcam), 6-phosphofructo-2-kinase/fructose-2,6-bisphosphatase 3 (PFKFB3; ab181861, dilution 1:1,000; Abcam), $\beta$-actin (3700, dilution 1:1,000; Cell Signaling Technology, Inc., Danvers, MA, USA) at $4^{\circ} \mathrm{C}$ overnight. Finally, the membranes were incubated with secondary antibodies conjugated with horseradish peroxidase for $1 \mathrm{~h}$ at room temperature. Immunoreactive materials were visualized by using Chemiluminescent Substrate kit (Pierce; Thermo Fisher Scientific, Inc.). The membranes were scanned and the sum optical density was 
quantitatively analyzed by Quantityone software (Bio-Rad Laboratories, Inc.).

Lactate concentration measurement. HUVECs were washed with ice cold PBS three times and split with RIPA for $20 \mathrm{~min}$ at $4^{\circ} \mathrm{C}$. Lysates was centrifuged and supernatant was analyzed by Amplite ${ }^{\mathrm{TM}}$ Colorimetricn L-Lactate Assay kit (AAT Bioquest, Inc., Sunnyvale, CA, USA) according to the manufacture's instruction. Lactate concentration in each well was normalized to total protein content by the Brandford assay.

ATP production measurement. $1 \times 10^{6}$ cells were harvested in $200 \mu 1$ PBS and cell lysate was achieved by sonication before the homogenate was centrifuged at $12,000 \mathrm{x}$ g for $5 \mathrm{~min}$. The intracellular ATP production was assessed by using an Enhanced ATP Assay kit (Beyotime Institute of Biotechnology) and the manufacturers' instructions were strictly followed. Luminescence was measured by a luminometer (Fluroskan Ascent FL; Thermo Fisher Scientific, Inc.). Data were normalized based on the protein concentration measured by the Brandford assay.

Measurement of mitochondrial membrane potential $(\Delta \psi m)$. Cells cultured in 6-well plates after indicated treatments were incubated with an equal volume of JC-1 staining solution $(5 \mu \mathrm{g} / \mathrm{ml})$ at $37^{\circ} \mathrm{C}$ for $20 \mathrm{~min}$ and rinsed twice with PBS. Mitochondrial membrane potential was monitored by determining the relative amounts of dual emissions from mitochondrial JC-1 monomers or aggregates using an Olympus fluorescent microscope under $488 \mathrm{~nm}$ laser excitation.

Moreover, the fluorescence intensity was detected with a flow cytometry (BD FACSCalibur; BD Biosciences, Franklin Lakes, NJ, USA). The wavelengths of excitation and emission were 514 and $529 \mathrm{~nm}$ for detection of monomeric form of JC-1. 585 and $590 \mathrm{~nm}$ were used to detect aggregation of JC-1. Mitochondrial depolarization is indicated by an increase in the green/red fluorescence intensity ratio.

Transmission electron microscope. HUVECs were harvested using trypsin-EDTA. After washing for three tomes with ice cold PBS, cells were fixed with $4 \%$ glutaraldehyde overnight at $4^{\circ} \mathrm{C}$. A specimen was cut into ultra-thin sections of $60-68 \mathrm{~nm}$. A JEM-1010 model transmission electron microscope (Japan Electron Optics Laboratory Company, Tokyo, Japan) was used to observe the ultra-microstructures of mitochondrial.

Mitochondrial pyruvate measurement. Mitochondrial pyruvate concentration was determined by pyruvate assay kit (BioVision, California, USA) according to the instructions. Briefly, $50 \mu \mathrm{l}$ working reagent and $50 \mu 1$ test or standard sample were mixed, and then added in 96-well plate. After 30 min's incubation at room temperature, the color intensity of the reaction product at $570 \mathrm{~nm}$ was read and recorded with a Microplate Reader (Infinite ${ }^{\circledR}$ M200; Tecan Ltd.). The results were normalized to the total cell numbers.

Statistical analysis. Data were presented as means \pm standard deviation. One-way analysis of variance was used for multiple comparisons by SPSS 19.0 (SPSS, Inc, Chicago, IL, USA).
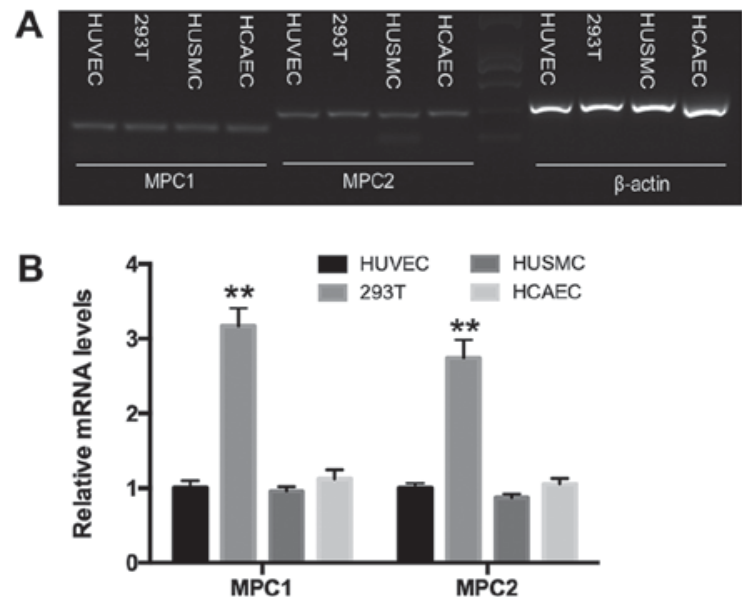

Figure 1. Determination of MPC1 and MPC2 expression. MPC1 and MPC2 mRNA expression in HUVECs, HUSMCs, HCAEC, 293T cells were detected by (A) RT-PCR and (B) qPCR. Data are expressed as mean \pm standard deviation, $n=3$. $^{* *} \mathrm{P}<0.01$ vs. HUVECs, HUSMC and HCAEC. MPC, mitochondrial pyruvate carrier; HUVEC, human umbilical vein endothelial cell; HUSMC, human umbilical vein smooth muscle cell; HCAEC, human coronary artery endothelial cell.

If there was a significant variation between treated groups, Tukey's post hoc test was applied. $\mathrm{P}<0.05$ was considered to indicate a statistically significant difference.

\section{Results}

MPC expression levels in different metabolic cell types. To evaluate whether MPC expression varies among different cell types, we examined the MPC levels in HUVECs, HCAECs, 293T cells, and HUSMCs. The former three cell types rely on glycolysis for energy production while HUSMCs rely on oxidation $(12,13)$. Using reverse transcription-polymerase chain reaction (RT-PCR), both $m p c 1$ and $m p c 2$ were shown to be expressed in HUVECs, HCAECs, HUSMCs, and 293T cells (Fig. 1A). qPCR demonstrated that, compared to the other cell types, MPC1 and MPC2 were highly expressed in 293T cells, a typical anaerobic cell type (13). No significant difference was observed in MPC expression among HUVECs, HCAECs, and HUSMCs (Fig. 1B).

The effect of hypoxia on lactate secretion and MPC expression in HUVECs. Lactate, generated from pyruvate by LDHA, is the final product of glycolysis. To determine the effect of hypoxia on this metabolic process, we measured the lactate levels as well as MPC expression upon exposure to hypoxia in HUVECs. An L-lactate acid assay revealed that the extracellular lactate concentration was increased under hypoxia for $24 \mathrm{~h}$ in HUVECs, while the levels of intracellular lactate were not significantly affected (Fig. 2A). This suggests that lactate efflux was upregulated under hypoxia. qPCR and Western blotting showed that the expression of MPC1 and MPC2 was downregulated under conditions of hypoxia lasting for $24 \mathrm{~h}$ and subsequently induced following re-oxygenation (Fig. 2B and C). Opa1 and Fis1, two key factors in mediating mitochondrial fusion and fission, were used as positive controls as they have been shown to be influenced by extracellular oxygen levels (14). As demonstrated 
A

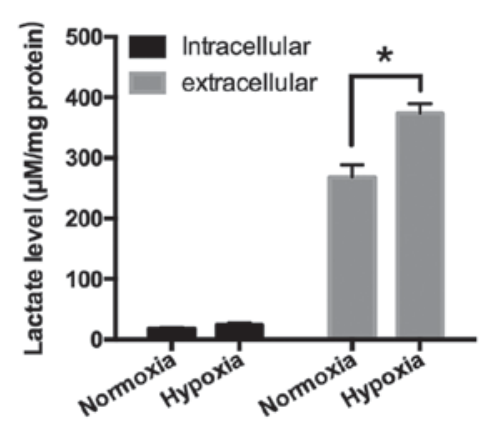

B

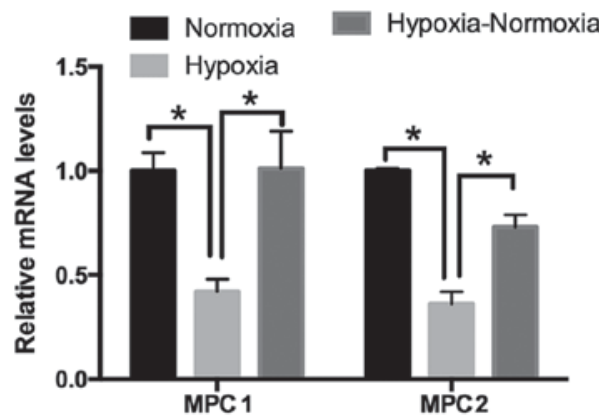

C

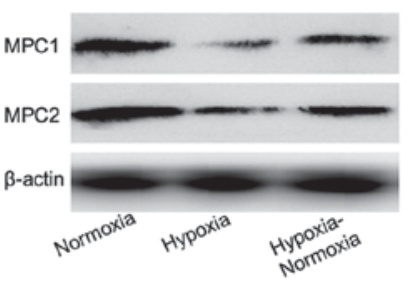

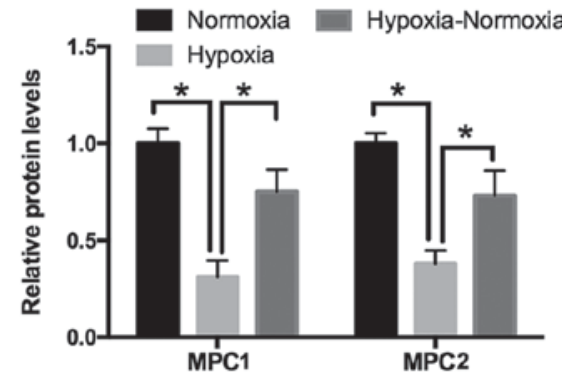

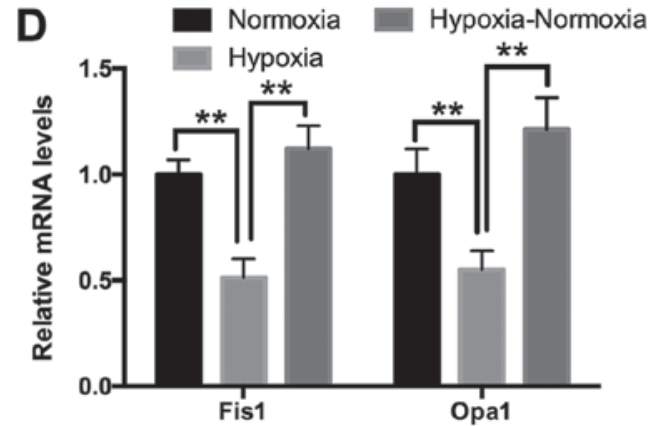

Figure 2. Hypoxia increased lactate secretion and decreased MPC in HUVECs. (A) Intracellular and extra cellular lactate concentration under hypoxia intervention for $24 \mathrm{~h}$. Lactate concentration in each well was normalized to total protein content. MPC1, MPC2 mRNA and protein were detected by (B) qPCR and (C) western blot analysis, respectively. $\beta$-actin was used for normalization. (D) Fis1 and Opa1 mRNA were detected by qPCR. The results are expressed as mean \pm standard deviation, $\mathrm{n}=3$. ${ }^{*} \mathrm{P}<0.05,{ }^{* *} \mathrm{P}<0.01$. MPC, mitochondrial pyruvate carrier; HUVEC, human umbilical vein endothelial cell.

in Fig. 2D, HUVECs exposed to hypoxia exhibited significantly increased mRNA levels of Opal and Fisl at $24 \mathrm{~h}$ post-treatment; further, these levels were increased following re-oxygenation for $24 \mathrm{~h}$.

Role of the MPC complex in hypoxia-induced lactate secretion and aerobic glycolysis in HUVECs. To evaluate whether hypoxia-induced lactate secretion was related to MPC function, MPC1 expression was silenced using an siRNA and MPC activity was inhibited using UK5099 (15). Western blotting revealed that the siRNA targeting MPC1 (siMPC1) reduced MPC1 levels specifically without affecting MPC2 expression (Fig. 3A). It was discovered that UK5099 was optimized to a final concentration of $40 \mu \mathrm{M}$ to effectively reduce pyruvate transportation into mitochondria (Fig. 3B). Both MPC1 silencing and treatment with UK5099 in HUVECs increased the extracellular lactate concentration significantly, indicating a higher glycolytic efflux, thus produced more lactic acid (Fig. 3C). In contrast, ATP production in samples treated with UK5099 or siMPC1 was significantly lower than that in control cells (Fig. 3D). Next, to determine how the inhibition of MPC affects glycolysis, the levels of the key glycolytic enzymes HK2, LDHA, and PFKFB3 were measured using Western blotting in HUVECs treated with or without siRNA or UK5099. As shown in Fig. 3E, exposure to hypoxia led to the upregulation of HK2, LDHA, and PFKFB3, suggesting that hypoxia promotes glycolysis. Similarly, treatment with UK5099 or MPC1 silencing resulted in a significant increase in the protein expression of HK2, LDHA, and PFKFB3 (Fig. 3E).

The effect of MPC inhibition on mitochondrial structure and the mitochondrial membrane potential $(\Delta \psi m)$ in HUVECs. During mitochondrial respiratory oxidation, energy generated by the electrochemical chain reaction is stored within the IMM. Changes in this energy generation process can impact the morphology of the organelle. To determine whether mitochondrial structure was affected by the inhibition of MPC, transmission electron microcopy was utilized to examine the mitochondrial morphology in HUVECs treated with UK5099 or siRNA. Compared with control treatment groups, MPC1 silencing or UK5099 treatment did not lead to significant alterations in mitochondrial structure (i.e., no mitochondrial swelling, pyknosis, and ambiguous cristae were observed; Fig. 4A).

The mitochondrial $\Delta \psi \mathrm{m}$, an important parameter reflecting mitochondrial function (16), can be measured using the fluorescent dye JC-1. JC-1 accumulates and aggregates in healthy mitochondria and can be visualized as red fluorescence. The treatment of HUVECs with carbonyl cyanide-m-chlorophenylhydrazone (CCCP), an OXPHOS inhibitor (17), resulted in an increase in green fluorescence, indicating a reduction in the mitochondrial $\Delta \psi \mathrm{m}$ (Fig. 4B). Similarly, the depletion of MPC1 using an siRNA or inhibition of MPC activity by UK5099 decreased the mitochondrial $\Delta \psi \mathrm{m}$ (Fig. 4B). Dissipation of the mitochondrial $\Delta \psi \mathrm{m}$ can also be represented by the ratio of aggregated to monomeric $\mathrm{JC}-1$, since the monomeric form of JC-1 re-localizes to the cytosol following its disassociation in mitochondria. Using flow cytometry, a significant amount of JC-1 aggregates was observed in the mitochondria of control cells (Fig. 4C). In contrast, cells treated with CCCP had a lower aggregated to monomeric JC-1 ratio (Fig. 4C). HUVECs treated with UK5099 or siMPC1 exhibited a reduced mitochondrial $\Delta \psi \mathrm{m}$ (Fig. 4C). Opal and Fis1 mediate mitochondrial fusion and fission, and are important for the maintenance of the organelle's function. qPCR revealed that the Opal and 
A

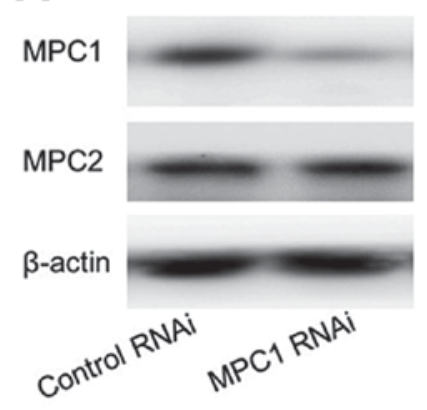

C
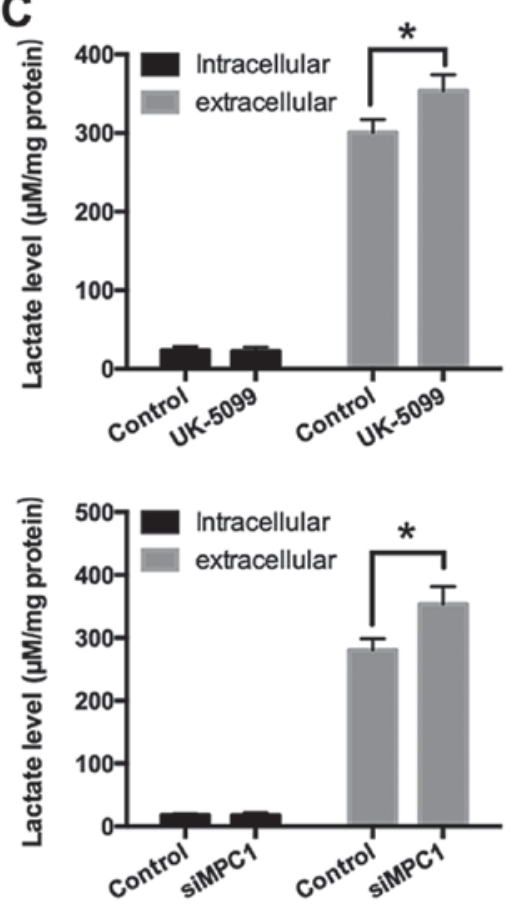

B

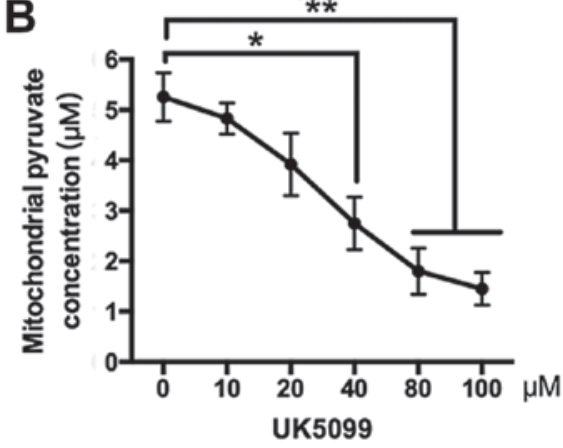

D

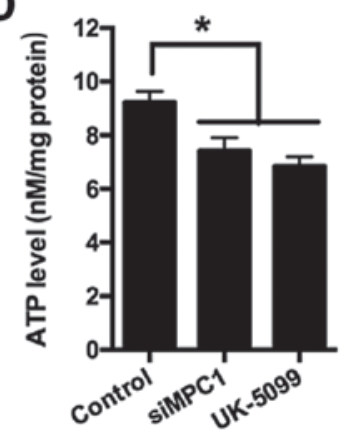

E
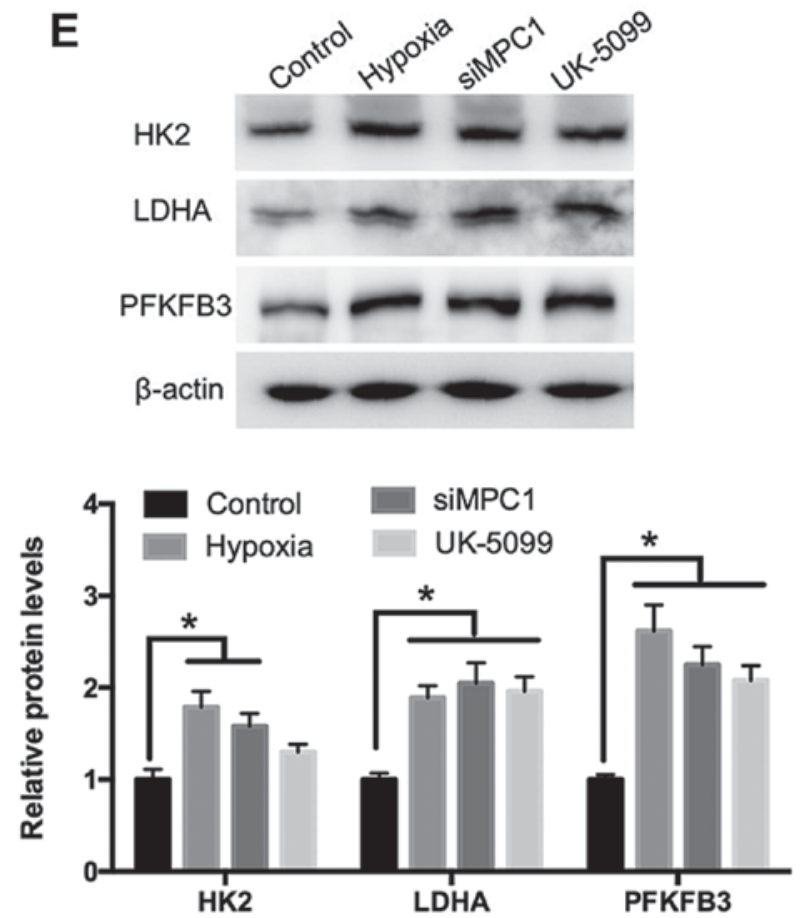

Figure 3. MPC contributed to hypoxia-induced lactate secretion and aerobic glycolysis in HUVECs. (A) The efficiency of MPC1 siRNA was verified using western blotting. $\beta$-actin was analyzed for a loading control. (B) Mitochondrial pyruvate concentration after the treatment of UK5099. (C) Intracellular and extra cellular lactate concentration at different interventions. Lactate concentration in each well was normalized to total protein content. (D) ATP concentration at different interventions. ATP concentration in each well was normalized to total protein content. (E) The protein level of HK2, LDHA and PFKFB3 were detected by western blot analysis. The $\beta$-actin serves as a loading control. The results are expressed as mean \pm standard deviation, $\mathrm{n}=3$. ${ }^{*} \mathrm{P}<0.05,{ }^{* *} \mathrm{P}<0.01$. MPC, mitochondrial pyruvate carrier; HUVEC, human umbilical vein endothelial cell; HK2, hexokinase II; LDHA, lactate dehydrogenase A; PFKFB3, 6-phosphofructo-2-kinase/fructose-2,6-bisphosphatase 3.

Fisl mRNA levels were reduced in MPC1-knockdown and UK5099-treated HUVECs (Fig. 4D), suggesting a role for MPC in mitochondrial function.

\section{Discussion}

A previous study showed that the MPC complex is a key regulator of glycolysis in tumor cells (11). Independent of the EC subtype, arterial, venous, lymphatic, and microvascular ECs rely heavily on glycolysis for energy production regardless of the abundance of oxygen (8). In this study, we found that hypoxia decreased MPC1 and MPC2 expression in HUVECs. This was correlated with an upregulation in the levels of glycolytic enzymes, leading to increased glycolysis and lactate secretion.

In contrast to other cell types with greater energy needs, there is a moderate number of mitochondria in ECs. The mitochondrial volume in these cells is only $2-6 \%$ of the total cellular volume, compared to $32 \%$ in cardiomyocytes and $28 \%$ in hepatocytes $(16,18,19)$. Interestingly, we found that HUVECs, HCAECs, and HUSMCs had similar transcript levels of $M P C 1$ and $M P C 2$, suggesting that mitochondrial volume may not be the deciding factor for MPC expression.

Lactate is generated through the metabolism of pyruvate by LDHA as the final step of glycolysis. The effect of hypoxia on EC metabolism remains poorly understood. Previous studies have suggested that hypoxia promotes glycolysis by upregulating the expression of glycolysis-promoting genes, stabilizing HIF-1 $\alpha$, inhibiting pyruvate dehydrogenase kinase and prolyl hydroxylase, and inducing lactate secretion (20). Recently, De Bock et al (8) demonstrated that glycolysis in ECs is modulated by the enzyme PFKFB3, an activator of phosphofructokinase 1 , which is a rate-limiting enzyme in glycolysis. Xu et al (12) later reported that PFKFB3 expression 
A

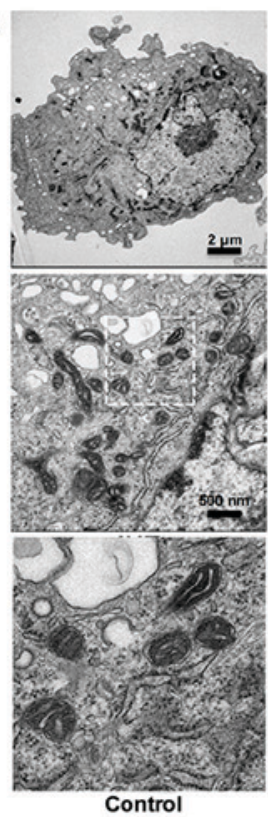

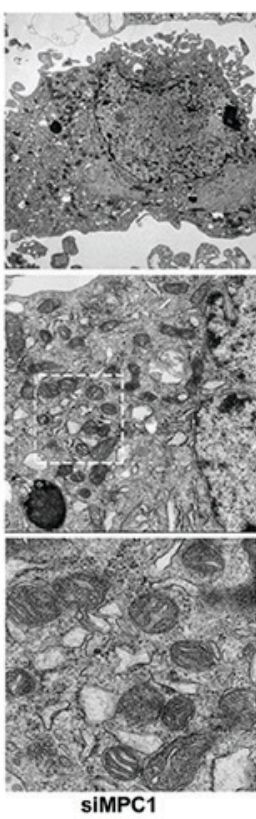

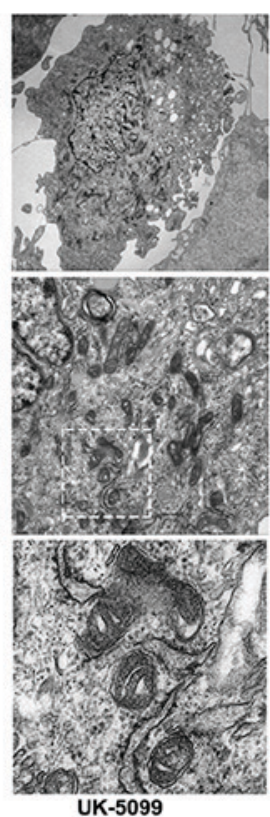

B
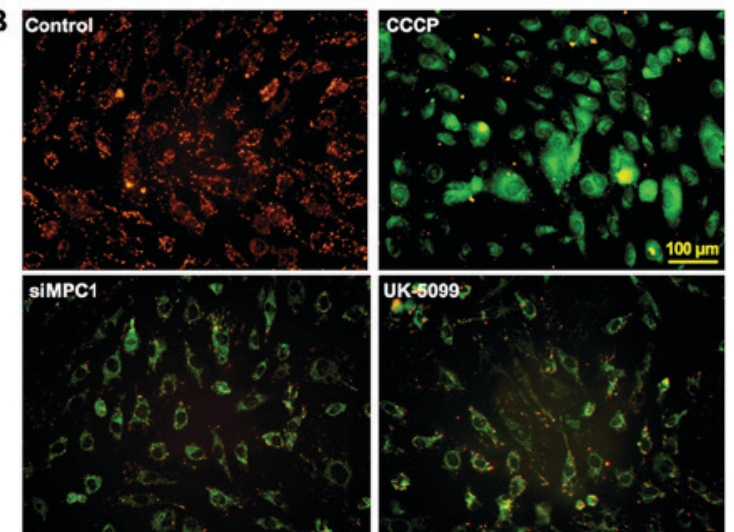

C

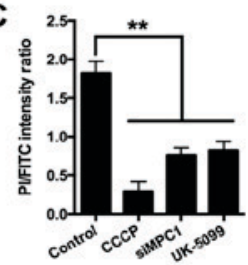

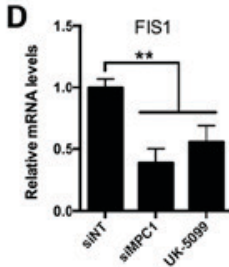

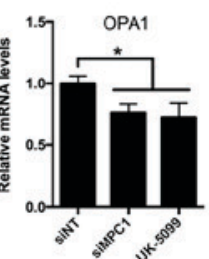

Figure 4. MPC inhibition decreased mitochondrial membrane potential ( $\Delta \psi \mathrm{m})$ but did not influence mitochondria structure in HUVECs. (A) The mitochondria morphology was determined at different interventions for $24 \mathrm{~h}$ by FEM. (B) The mitochondrial membrane potential was determined by JC-1 staining. Red fluorescence represents the mitochondrial aggregate form of JC-1, indicating intact mitochondrial membrane potential. Green fluorescence represents the monomeric form of JC-1, indicating dissipation of mitochondrial membrane potential. CCCP was the positive control. Scale bar is $50 \mu \mathrm{M}$. (C) The ratio of red (PI) to green (FITC) fluorescence were checked by flow cytometry. (D) Fis1 and Opa1 mRNA were detected by qPCR. The results are expressed as mean \pm standard deviation, $\mathrm{n}=3 .{ }^{*} \mathrm{P}<0.05,{ }^{* *} \mathrm{P}<0.01$. MPC, mitochondrial pyruvate carrier; HUVEC, human umbilical vein endothelial cell; $\mathrm{CCCP}$, carbonyl cyanide-m-chlorophenylhydrazone; PI, propidium iodide; FITC, fluorescein isothiocyanate.

was increased upon exposure to hypoxia in ECs. Consistent with these findings, our data show that HUVECs upregulated lactate secretion by increasing the levels of the glycolytic enzymes HK2, LDHA, and PFKFB3 under hypoxic conditions.

Hypoxia signaling can induce a shift from other forms of energy production to glycolysis-dependent means (21). In $\mathrm{ECs}$, in which glycolysis is the main form of energy generation, hypoxia may have a more profound impact on other metabolic pathways, including mitochondrial OXPHOS. Low oxygen levels regulate glycolysis-promoting genes while decreasing pyruvate metabolism (22). Given the important role of the MPC complex in pyruvate metabolism, alterations in the expression of MPC could have a significant effect on cells. We found that the expression of MPC1 and MPC2 was decreased when the oxygen level was low, and that their expression levels recovered following re-oxygenation. This suggests that the function of the MPC complex is regulated by oxygen concentrations. The transportation of pyruvate into mitochondria by the MPC complex is the first step in mitochondrial OXPHOS; however, how this process may affect glycolysis is unclear. Li et al (11) reported that blocking pyruvate transportation into mitochondria using the MPC blocker UK5099 attenuated mitochondrial OXPHOS and triggered aerobic glycolysis in esophageal squamous cell carcinomas. As pyruvate plays a central role in glucose and lipid metabolism, we speculated that dysregulation of MPC function may alter the expression of other metabolic genes apart from those involved in OXPHOS. Indeed, treatment with UK5099 or MPC1 silencing upregulated the glycolytic enzymes HK2, LDHA, and PFKFB3 in HUVECs. However, the mechanism of MPC inhibition-induced glycolytic enzymes expression is unclear in ECs. During hypoxia, hypoxia-inducible factor (HIF) signaling regulates multiple aspects of ECs metabolism. Stabilization of HIF1 $\alpha$ results in a switch from OXPHOS towards glycolysis by binding to a hypoxia response element (HRE) in the promoter and thus upregulating glycolysis-promoting genes, such as HK, glyceraldehyde-3-phosphate dehydrogenase, LDHA and pyruvate dehydrogenase kinase 1 (PDK1) (22). Furthermore, lactate production from glycolysis flux can further stabilize HIF1 $\alpha$. In the present study, we found that treatment with UK5099 or MPC1 silencing increased lactate secretion. Therefore, we consider that MPC inhibition increases glycolytic enzymes via both directly and indirectly stabilize HIF1 $\alpha$. A future work will be performed to fully understand the mechanism.

Due to an insufficient oxygen supply, mitochondrial OXPHOS is inhibited. ATP levels are also decreased during the initial phase of hypoxia as a result of reduced glycolysis. As glycolysis recovers, the production of ATP increases as long as sufficient amounts of substrates are available (23), while the concentration of lactate rises steadily. Our data reveal a significant decrease in ATP synthesis and increase in lactate production in HUVECs treated with UK5099 or siMPC1. This suggests that impaired mitochondrial pyruvate transport could drive glycolysis to promote lactate secretion in HUVECs.

Mitochondrial function is closely related to the amount of pyruvate entering mitochondria (16). In our study, we showed that the mitochondrial $\Delta \psi \mathrm{m}$ dropped when MPC1 was silenced or when MPC function was inhibited, suggesting that hypoxia-induced mitochondrial dysfunction is related to MPC 
expression and/or activity. Interestingly, little change was detected in mitochondrial morphology. However, we cannot rule out the possibility that the inhibition of MPC could induce changes in organelle structure if treatment was prolonged (> $24 \mathrm{~h})$.

Fission and fusion mechanisms regulate mitochondrial morphology and apoptosis (14). Fission frequency is determined by the levels of Fis1, which localizes to the outer mitochondrial membrane. Fusion frequency is influenced by Opa1 levels, which are known to be associated with the IMM (24). It has been shown that the depletion of Opal leads to a reduction in mitochondrial $\Delta \psi \mathrm{m}$, while fragmentation of the mitochondrial network by Fis1 leads to cytochrome c release (14). Other studies have indicated that hypoxia/re-oxygenation results in a significant reduction in Opal levels and upregulation of Fis1 in cardiomyocytes (25) and hippocampal neurons (26). However, Chitra and Boopathy (27) reported that hypobaric hypoxia modulated mitochondrial dynamics by decreasing Fis1 in rat lung cells. Our data demonstrate that Opal and Fis1 mRNA levels were reduced after MPC1 knockdown or UK5099 treatment. Additional experimental investigations are needed to better understand the role of the MPC complex in mitochondrial fission and fusion.

In conclusion, we found that the MPC complex may play an essential role in hypoxia-induced glycolysis and lactate secretion in HUVECs. The depletion of MPC1 or inhibition of the MPC complex leads to increased lactate production, potentially by upregulating glycolytic enzymes and therefore promoting glycolysis. This study is the first attempt to link hypoxia to the MPC complex. It reveals MPC as a potential target for the treatment of hypoxia-related injury to ECs, including acute myocardial infarction and pulmonary hypertension. Limiting glycolysis decreased endothelial sprouting (28), showing the role of glycolysis on angiogenesis. As we known, angiogenesis has close relationship with cancer. It is thus tempting to speculate that MPC may be a novel target for the prevention and treatment of cancer. In conclusion, we demonstrated that hypoxia can induce lactate secretion and glycolytic efflux by downregulating MPC levels. Our study provided the evidence that MPC complex may play an essential role in hypoxia-induced glycolysis and lactate secretion in HUVECs. MPC might be a novel treatment target for hypoxia-induced EC injury, such as acute myocardial infarction and pulmonary hypertension.

\section{Acknowledgements}

Not applicable.

\section{Funding}

The present work was supported by Chengchun Tang (NSF nos. 81670237 and 81370225) and Dong Wang (the Fundamental Research Funds for the Central Universities, no. 2242018K40159).

\section{Availability of data and materials}

The datasets used during the present study are available from the corresponding author on reasonable request.

\section{Authors' Contributions}

DW, QW and CT designed the research. DW, QW, BZ and BL performed the experiments. DW, QW, GY and YQ analyzed the data. DW and CT wrote the manuscript. All authors reviewed and edited the manuscript.

\section{Ethics approval and consent to participate}

Not applicable.

\section{Consent for publication}

Not applicable.

\section{Competing interests}

The authors declare that they have no competing interests.

\section{References}

1. Herzig S, Raemy E, Montessuit S, Veuthey JL, Zamboni N, Westermann B, Kunji ER and Martinou JC: Identification and functional expression of the mitochondrial pyruvate carrier. Science 337: 93-96, 2012.

2. Bricker DK, Taylor EB, Schell JC, Orsak T, Boutron A, Chen YC, Cox JE, Cardon CM, Van Vranken JG, Dephoure N, et al: A mitochondrial pyruvate carrier required for pyruvate uptake in yeast, Drosophila, and humans. Science 337: 96-100, 2012.

3. Schell JC, Wisidagama DR, Bensard C, Zhao H, Wei P, Tanner J, Flores A, Mohlman J, Sorensen LK, Earl CS, et al: Control of intestinal stem cell function and proliferation by mitochondrial pyruvate metabolism. Nat Cell Biol 19: 1027-1036, 2017.

4. Divakaruni AS, Wallace M, Buren C, Martyniuk K, Andreyev AY, Li E, Fields JA, Cordes T, Reynolds IJ, Bloodgood BL, et al: Inhibition of the mitochondrial pyruvate carrier protects from excitotoxic neuronal death. J Cell Biol 216: 1091-1105, 2017.

5. Vigueira PA, McCommis KS, Hodges WT, Schweitzer GG, Cole SL, Oonthonpan L, Taylor EB, McDonald WG, Kletzien RF, Colca JR and Finck BN: The beneficial metabolic effects of insulin sensitizers are not attenuated by mitochondrial pyruvate carrier 2 hypomorphism. Exp Physiol 102: 985-999, 2017.

6. McCommis KS, Hodges WT, Brunt EM, Nalbantoglu I, McDonald WG, Holley C, Fujiwara H, Schaffer JE, Colca JR and Finck BN: Targeting the mitochondrial pyruvate carrier attenuates fibrosis in a mouse model of nonalcoholic steatohepatitis. Hepatology 65: 1543-1556, 2017.

7. Bender T and Martinou JC: The mitochondrial pyruvate carrier in health and disease: To carry or not to carry? Biochim Biophys Acta 1863: 2436-2442, 2016.

8. De Bock K, Georgiadou M, Schoors S, Kuchnio A, Wong BW, Cantelmo AR, Quaegebeur A, Ghesquière B, Cauwenberghs S, Eelen G, et al: Role of PFKFB3-driven glycolysis in vessel sprouting. Cell 154: 651-663, 2013.

9. Bachetti T and Morbidelli L: Endothelial cells in culture: A model for studying vascular functions. Pharmacol Res 42: 9-19, 2000.

10. Patterson JN, Cousteils K, Lou JW, Manning Fox JE, MacDonald PE and Joseph JW: Mitochondrial metabolism of pyruvate is essential for regulating glucose-stimulated insulin secretion. J Biol Chem 289: 13335-13346, 2014.

11. Li Y, Li X, Kan Q, Zhang M, Li X, Xu R, Wang J, Yu D, Goscinski MA, Wen JG, et al: Mitochondrial pyruvate carrier function is negatively linked to Warburg phenotype in vitro and malignant features in esophageal squamous cell carcinomas. Oncotarget 8: 1058-1073, 2017.

12. Xu Y, An X, Guo X, Habtetsion TG, Wang Y, Xu X, Kandala S, Li Q, Li H, Zhang C, et al: Endothelial PFKFB3 plays a critical role in angiogenesis. Arterioscler Thromb Vasc Biol 34: 1231-1239, 2014. 
13. Yu Z, Zhao X, Huang L, Zhang T, Yang F, Xie L, Song S, Miao P, Zhao L, Sun X, et al: Proviral insertion in murine lymphomas 2 (PIM2) oncogene phosphorylates pyruvate kinase M2 (PKM2) and promotes glycolysis in cancer cells. J Biol Chem 288: 35406-35416, 2013.

14. Lee YJ, Jeong SY, Karbowski M, Smith CL and Youle RJ: Roles of the mammalian mitochondrial fission and fusion mediators Fis1, Drp1, and Opa1 in apoptosis. Mol Biol Cell 15: 5001-5011, 2004.

15. Hildyard JC, Ammälä C, Dukes ID, Thomson SA and Halestrap AP: Identification and characterisation of a new class of highly specific and potent inhibitors of the mitochondrial pyruvate carrier. Biochim Biophys Acta 1707: 221-230, 2005.

16. Tang X, Luo YX, Chen HZ and Liu DP: Mitochondria, endothelial cell function, and vascular diseases. Front Physiol 5: 175, 2014.

17. Xiao B, Deng X, Lim GGY, Xie S, Zhou ZD, Lim KL and Tan EK: Superoxide drives progression of Parkin/PINK1-dependent mitophagy following translocation of Parkin to mitochondria. Cell Death Dis 8: e3097, 2017.

18. Dromparis P and Michelakis ED: Mitochondria in vascular health and disease. Annu Rev Physiol 75: 95-126, 2013.

19. Kluge MA, Fetterman JL and Vita JA: Mitochondria and endothelial function. Circ Res 112: 1171-1188, 2013.

20. Wong BW, Marsch E, Treps L, Baes M and Carmeliet P: Endothelial cell metabolism in health and disease: Impact of hypoxia. EMBO J 36: 2187-2203, 2017.

21. Tretyakov AV and Farber HW: Endothelial cell tolerance to hypoxia. Potential role of purine nucleotide phosphates. J Clin Invest 95: 738-744, 1995
22. Schofield CJ and Ratcliffe PJ: Oxygen sensing by HIF hydroxylases. Nat Rev Mol Cell Biol 5: 343-354, 2004.

23. Jezek P and Plecitá-Hlavatá L: Mitochondrial reticulum network dynamics in relation to oxidative stress, redox regulation, and hypoxia. Int J Biochem Cell Biol 41: 1790-1804, 2009.

24. Olichon A, Baricault L, Gas N, Guillou E, Valette A, Belenguer P and Lenaers G: Loss of OPA1 perturbates the mitochondrial inner membrane structure and integrity, leading to cytochrome $\mathrm{c}$ release and apoptosis. J Biol Chem 278: 7743-7746, 2003.

25. Yu J, Wu J, Xie P, Maimaitili Y, Wang J, Xia Z, Gao F, Zhang $\mathrm{X}$ and Zheng $\mathrm{H}$ : Sevoflurane postconditioning attenuates cardiomyocyte hypoxia/reoxygenation injury via restoring mitochondrial morphology. PeerJ 4: e2659, 2016.

26. Zhao L, Li S, Wang S, Yu N and Liu J: The effect of mitochondrial calcium uniporter on mitochondrial fission in hippocampus cells ischemia/reperfusion injury. Biochem Biophys Res Commun 461: 537-542, 2015.

27. Chitra L and Boopathy R: Adaptability to hypobaric hypoxia is facilitated through mitochondrial bioenergetics: An in vivo study. Br J Pharmacol 169: 1035-1047, 2013.

28. Boeckel JN, Derlet A, Glaser SF, Luczak A, Lucas T, Heumüller AW, Krüger M, Zehendner CM, Kaluza D, Doddaballapur A, et al: JMJD8 regulates angiogenic sprouting and cellular metabolism by interacting with pyruvate kinase M2 in endothelial cells. Arterioscler Thromb Vasc Biol 36: 1425-1433, 2016 